\title{
A comparative study on the short-term clinical efficacy of the modified laparoscopic uterine comminution technique and traditional methods
}

\author{
XIAOJUN SHI ${ }^{1}$, LIBING SHI ${ }^{2}$ and SONGYING ZHANG ${ }^{2}$ \\ ${ }^{1}$ Department of Gynecology, The First Affiliated Hospital of Jiaxing University, Jiaxing, Zhejiang 314001; \\ ${ }^{2}$ Assisted Reproduction Unit, Department of Obstetrics and Gynecology, Sir Run Run Shaw Hospital, \\ School of Medicine, Zhejiang University, Hangzhou, Zhejiang 310016, P.R. China
}

Received April 28, 2019; Accepted December 13, 2019

DOI: $10.3892 / \mathrm{mco} .2020 .1982$

\begin{abstract}
To assess the value of the modified laparoscopic uterine comminution technique in laparoscopic uterine surgery, a total of 82 cases of laparoscopic myomectomy were divided into the traditional group and modified group, according to a random number table. During the same period, 92 patients who underwent laparoscopic hysterectomy were divided into the conventional group and the modified group, according to a random number table. The patients in the conventional group and modified group who underwent laparoscopic uterine fibroid removal showed no significant differences in the operation time, blood loss or average hospitalization $(\mathrm{P}>0.05)$. There was no significant difference in the operative time or average length of hospital stay between patients in the conventional group and modified group who underwent laparoscopic hysterectomy $(\mathrm{P}>0.05)$. In laparoscopic myomectomy, the fibroid specimens were placed in a self-made specimen bag for modified uterine comminution. In laparoscopic hysterectomy, the whole uterus specimen was placed in a self-made specimen bag and viewed from the vagina. The improved comminution technique is simple and feasible, does not increase the operation time or length of hospitalization, and has value for clinical use.
\end{abstract}

\section{Introduction}

Laparoscopic minimally invasive surgery is widely used in gynecological operations. Gynecological laparoscopy is a minimally invasive diagnostic and treatment technology that integrates modern gynecological surgery and endoscopic

Correspondence to: Dr Xiaojun Shi, Department of Gynecology, The First Affiliated Hospital of Jiaxing University, 1882 Central South Road, Jiaxing, Zhejiang 314001, P.R. China

E-mail: shixiaojun81@126.com

Key words: laparoscopic myomectomy, laparoscopic hysterectomy, modified laparoscopic uterine smashing technique diagnosis and treatment. It has thus become one of the most commonly used techniques in the field of gynecology. Uterine fibroids are common benign tumors of the female genital organs and the prevalence has been reported as high as 20 to $40 \%(1,2)$. The widespread application of pulverizers allows for removal of uterine fibroids under laparoscopy, which better reflects the superiority of minimally invasive techniques. However, preoperative diagnosis of uterine fibroids and uterine sarcoma is very difficult. The incidence of uterine fibroid sarcoma is approximately 0.03 to $1.00 \%$ (3). Despite the dramatic advances in imaging technology, diagnostic criteria for preoperative suspicious leiomyosarcoma are still lacking. The direct use of a pulverizer under laparoscopic surgery may cause the tumor to spread in the abdominal cavity, leading to the need for secondary surgery and affecting the prognosis (4). To prevent this, after laparoscopic removal of uterine fibroids, the tumor is placed in a specimen bag, and the modified uterine fibroids are comminuted in the bag. If laparoscopic hysterectomy is performed, the isolated uterus is large and difficult to remove from the vagina at one time. We use a self-made specimen bag to facilitate smashing of the uterus in the lower pocket of the vagina. This procedure is described in the present report.

\section{Patients and methods}

General information. We identified 82 cases of laparoscopic myomectomy in our hospital from April 2017 to October 2018. All patients were diagnosed as intermuscular myoma and subserosal fibroids, according to preoperative examination. The study protocol was approved by the Institutional The First Affiliated Hospital of Jiaxing University (2017-192), and all eligible patients provided written informed consent prior to treatment. The inclusion criteria were as follows: i) B type-Ultrasound (transvaginal ultrasound) indicated uterine fibroids with a single uterine fibroid greater than $4.0 \mathrm{~cm}$ in diameter. ii) For patients with uterine fibroids, preoperative examination indicated dysplasia in the endometrium, a B type-ultrasound was performed on the fifth day of menstruation. Diagnostic curettage or hysteroscopy with diagnostic curettage will be performed for those whose endometrium 
was still abnormal. Patients can only be recruited when there was no endometrial cancer showed in the postoperative pathology. iii) Patients with rapid growth of uterine fibroids in short period should receive MRI to exclude the neoplasm before they can be recruited. The exclusion criteria included: i) Preoperative examination suggested uterine fibroids or endometerial canceration. ii) Patients that the fibroids were found degenerated during the intraoperative stripping and the consistency was abnormal, swicthed to receivethe laparatomic surgery. With approval of the ethics committee, 42 cases were randomly selected for the study. The modified uterine smashing technique was used in the experiment. Patients with other disease conditions were most common with cardiovascular disease and metabolic disorders, including 3 patients (7.1\%) with hypertension, 2 patients $(4.8 \%)$ with diabetes, and 1 patient with chronic bronchitis $(2.4 \%)$; 1 patient $(2.4 \%)$ with two or more complications. Postoperative pathologic examination confirmed 32 cases of uterine leiomyoma, 9 cases of uterine leiomyoma with degeneration and abundant cells, 1 case of uterine mucinous myoma. The remaining 40 patients underwent routine surgery without specimen bags. Including 2 patients $(5.0 \%)$ had hypertension, and 1 patient $(2.5 \%)$ had diabetes. Postoperative pathologic examination confirmed 34 cases of uterine leiomyoma and 6 cases of uterine leiomyoma with degeneration and abundant cells. There were no significant differences in the baseline data between the two groups $(\mathrm{P}>0.05)$. There were also no significant differences in the operation time, blood loss, or average hospital stay between the two groups $(\mathrm{P}>0.05$; Tables I and II).

We identified 92 patients who underwent laparoscopic hysterectomy with difficulty in removing the uterus directly from the vagina from April 2017 to October 2018 in our hospital. The study protocol was approved by the Institutional The First Affiliated Hospital of Jiaxing University (2017-192), and all eligible patients provided written informed consent prior to treatment. The inclusion criteria were as follows: i) According to the indications of hysteretomy. ii) If there were indications for removal of uterus and the preoperative examination showed abnormalities in the endometrium, a B type-Ultrasound was performed on the fifth day of menstruation. Diagnostic curettage or hysteroscopy with diagnostic curettage will be performed for those whose endometrium was still abnormal. Patients can only be recruited when there was no endometrial cancer showed in the postoperative pathology. The exclusion criteria included: The posibility of malignancy cannot be excluded and the malignancy was highly suspected, considering the uterus was usually large, a laparotomic surgery was needed and the patient should be excluded from the experiment. With approval of the ethics committee, 49 patients were randomly selected for inclusion in the study. Intraoperative self-made specimen bags were used for the modified uterine smashing technique. Patients with other disease conditions were most common with cardiovascular disease and metabolic disorders, including 5 patients $(10.2 \%)$ with hypertension, 4 patients $(8.2 \%)$ with diabetes, and 2 patient with chronic bronchitis $(4.1 \%)$; 3 patient $(6.1 \%)$ with two or more complications. There were 43 cases of laparoscopic uterine double salpingectomy and 6 cases of laparoscopic uterine bilateral adnexectomy. In total, 27 cases of uterine multiple leiomyoma were confirmed by postoperative pathology, including 4 cases of uterine leiomyoma with degeneration and abundant cells, 18 cases of myopathy, 3 cases of endometrial atypical hyperplasia, and 1 case of endometrial cancer. The patient was a perimenopausal woman, who was diagnosed with cervical intraepithelial neoplasia III and received cervical conization. The preoperative examination did not indicate abnormal endometrium. Because the patient strongly requested to remove the uterus, so a minimally invasive surgery was performed. Postoperative pathological accidentally showed endometrial malignancy. The remaining 43 patients underwent routine surgery without specimen bags. Including 6 patients $(14.0 \%)$ with hypertension, 4 patients $(9.3 \%)$ with diabetes, and 1 patient with chronic bronchitis $(2.3 \%)$; 4 patient $(9.3 \%)$ with two or more complications. Postoperative pathologic examination confirmed 31 cases of uterine leiomyoma, including 3 cases of uterine leiomyoma with degeneration and abundant cells, 10 cases of myopathy, 2 cases of endometrial atypical hyperplasia. There were no significant differences in the baseline data between the two groups $(\mathrm{P}>0.05)$. There were also no significant differences in the uterine weight, operative time, or average length of hospital stay between the two groups (P>0.05; Tables III and IV).

Specimen bag specifications. The width of our hospital's disposable laparoscopic sheath (disposable suction bag) is $20 \mathrm{~cm}$, and the end with the white line is selected as the head end of the specimen bag. The length must be larger than the diameter of the fibroids (generally approximately $10-15 \mathrm{~cm}$ ). After determining the length, the bottom of the specimen bag is knotted or tied with silk thread (Fig. 1).

Operation of specimen bag during uterine myomectomy. After establishment of general anesthesia, the laparoscopic instrument was appropriately positioned and the pre-made specimen bag was placed into the abdominal cavity through a $10-\mathrm{mm}$ trocar. The bag was then placed in the right fossa iliaca and the mouth of the bag was opened. Laparoscopic myomectomy was then routinely performed. After the fibroids had been completely removed, the fibroid specimens were placed directly into the specimen bag. The surgical wound was routinely sutured, and the $10-\mathrm{mm}$ puncture hole in the left lower abdomen was enlarged to $15 \mathrm{~mm}$. The end of the specimen bag with the white line was then placed outside the abdominal cavity, and a metal trocar was placed in the pulverizer. The head end of the specimen bag was fixed and sealed on the trocar by a white wire, and the inflation port on the trocar was connected to the pneumoperitoneum tube and opened for inflation until the laparoscopic direct-view specimen bag bulged and the line of sight was good (Figs. 2A and 3A). (If the trocar of the pulverizer has no inflation port, the pneumoperitoneum tube can be placed into the abdominal cavity together with the trocar). After the specimen bag was fixed in the same way, the pneumoperitoneum tube was used to inflate the specimen bag (Figs. 2B and 3B). After successful inflation of the specimen bag, the fibroids were pulverized within the specimen bag using the pulverizer under direct vision in the abdominal cavity, because the laparoscopic mirror is attached to the specimen bag, the line of sight is slightly blurred, but does not affect the operation (Fig. 3C-H). During this process, the specimen can be pulverized to $<1.5 \mathrm{~cm}$ (i.e., the size of the incision), and the 
Table I. Comparison of general basic data between the two groups in laparoscopic myomectomy.

\begin{tabular}{|c|c|c|c|}
\hline Parameter & Traditional group $(n=40)$ & Improved group $(n=42)$ & P-value \\
\hline Age, years & $42.77 \pm 6.25$ & $42.19 \pm 5.18$ & 0.645 \\
\hline BMI, $\mathrm{kg} / \mathrm{m}^{2}$ & $25.57 \pm 3.09$ & $25.27 \pm 3.48$ & 0.678 \\
\hline Number of caesarean section operations in the past & $0.83 \pm 0.64$ & $0.81 \pm 0.71$ & 0.917 \\
\hline Number of abdominal surgeries in the past & $1.08 \pm 0.76$ & $1.07 \pm 0.84$ & 0.984 \\
\hline Average diameter of uterine fibroids, $\mathrm{cm}$ & $7.43 \pm 2.10$ & $7.33 \pm 1.54$ & 0.822 \\
\hline Number of uterine fibroids & $1.33 \pm 0.57$ & $1.33 \pm 0.61$ & 0.949 \\
\hline
\end{tabular}

Table II. Comparison of surgical data between the two groups in laparoscopic myomectomy.

\begin{tabular}{lccc}
\hline Parameter & Traditional group $(\mathrm{n}=40)$ & Improved group $(\mathrm{n}=42)$ & P-value \\
\hline Operation time, min & $70.10 \pm 16.87$ & $71.40 \pm 17.71$ & 0.734 \\
Intraoperative blood loss, ml & $71.78 \pm 29.00$ & $68.38 \pm 29.94$ & 0.603 \\
Average hospital stays, days & $4.08 \pm 0.35$ & $4.05 \pm 0.22$ & 0.669 \\
\hline
\end{tabular}

Table III. Analysis of surgical data in laparoscopic hysterectomy.

\begin{tabular}{|c|c|c|c|}
\hline Parameter & Traditional group $(n=43)$ & Improved group $(\mathrm{n}=49)$ & P-value \\
\hline Age, years & $49.30 \pm 2.20$ & $49.51 \pm 2.33$ & 0.662 \\
\hline $\mathrm{BMI}, \mathrm{kg} / \mathrm{m}^{2}$ & $25.63 \pm 2.96$ & $26.08 \pm 3.62$ & 0.523 \\
\hline Number of caesarean surgeries in the past & $0.81 \pm 0.63$ & $0.78 \pm 0.69$ & 0.781 \\
\hline Number of abdominal surgeries in the past & $1.07 \pm 0.77$ & $1.02 \pm 0.80$ & 0.765 \\
\hline
\end{tabular}

Table IV. Comparison of surgical data between the two groups.

\begin{tabular}{lccc}
\hline Parameter & Traditional group $(\mathrm{n}=43)$ & Improved group $(\mathrm{n}=49)$ & P-value \\
\hline Uterine weight, $g$ & $324.79 \pm 128.43$ & $372.31 \pm 118.16$ & 0.068 \\
Operation time, min & $93.98 \pm 35.76$ & $95.02 \pm 25.40$ & 0.872 \\
Average hospital stays, days & $4.81 \pm 0.73$ & $4.76 \pm 0.56$ & 0.664 \\
\hline
\end{tabular}

remaining specimen fragments can be taken out together with the specimen bag from the abdominal cavity.

Operation of specimen bag during hysterectomy. After establishment of general anesthesia, routine laparoscopic hysterectomy was performed. The vaginal wall was completely disconnected via laparoscopy, than the self-made specimen bag which was from the umbilical trocar, was all placed in the abdominal cavity. All the uterus was placed into the specimen bag. The end of the specimen bag with the white line was tightened at the mouth of the uterus outside the uterus cup, and the end of the bag with the white line was removed from the vagina. After the mouth of the bag was outside the vaginal opening, the knot was opened and the uterus was removed. The uterus was then smashed under gross vision outside the vaginal opening (Fig. 4). The uterus was then taken out together with the specimen bag. When the specimen was taken in the vagina, the patient was changed to the supine position, and the intra-abdominal inflation was stopped.

Statistical analysis. All statistical analyses were performed using SPSS 21.0 software (IBM Corp.). Quantitative data are expressed as the mean \pm standard deviation, and the t-test was used for comparison between groups. Count data are expressed as a percentage. $\mathrm{P}<0.05$ was considered to indicate a statistically significant difference.

\section{Results}

Results and complications. Using the self-made specimen bag, the fibroids in the abdominal cavity were crushed and the uterus was smashed and removed under the vaginal opening. 


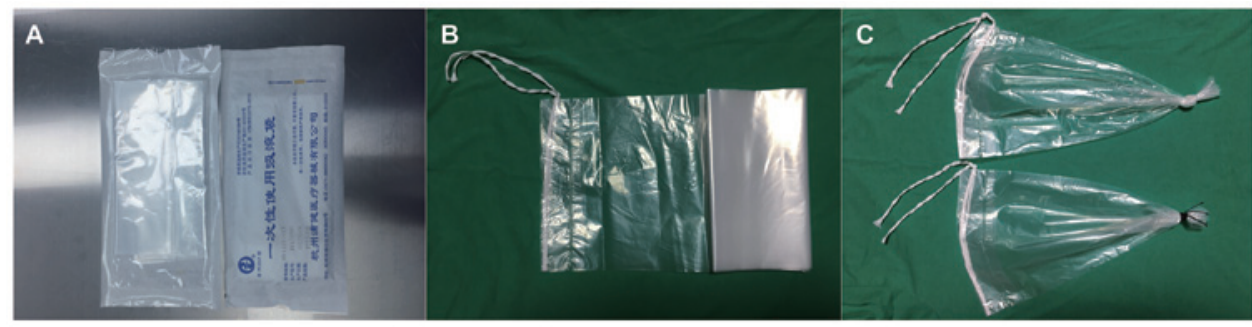

Figure 1. Laparoscopic sheath. (A) Scope of the 20x200 cm laparoscopic sheath. (B) Use one end with a white line as the head of the specimen bag. (C) Bottom of the specimen bag is knotted or tied with silk thread.
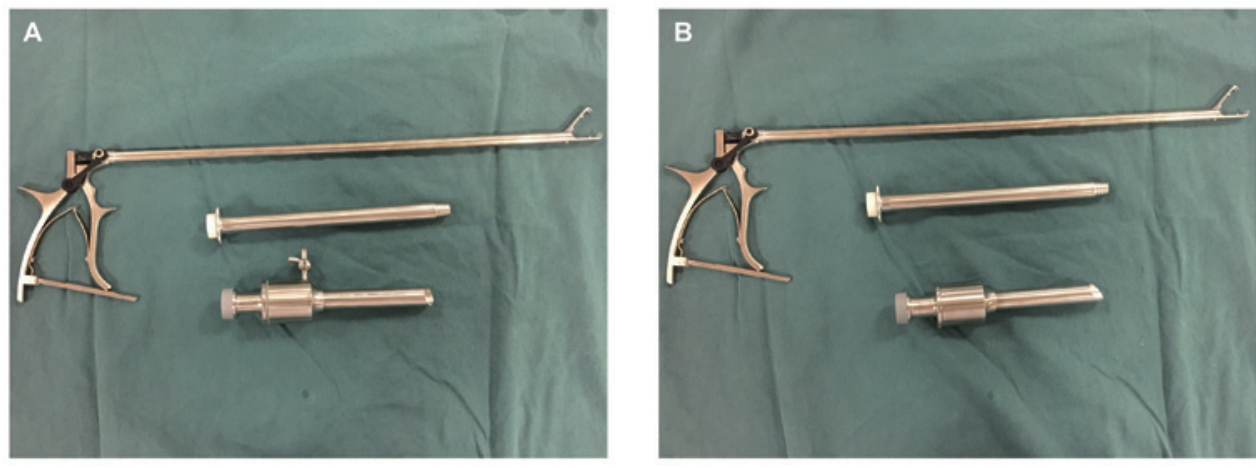

Figure 2. Crusher matching metal trocar. (A) Metal trocar with inflation port. (B) Metal trocar without inflation port.
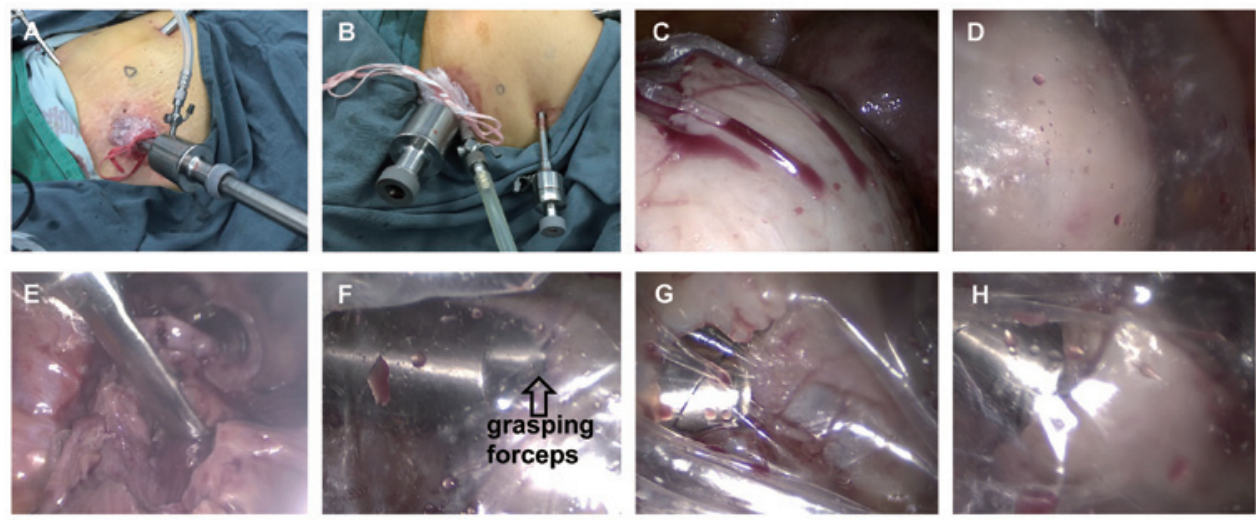

Figure 3. Modified laparoscopic uterine comminution technique. (A) Inflatable port trocar directly connected to the gas tube. (B) Trocar without inflation port uses a Veress needle to connect the gas tube. (C) Laparoscopic specimen bag before inflation. (D) Laparoscopic specimen bag after inflation. (E) Grab the specimen directly in the specimen bag. (F) Grasping forceps hiden in the cutter head to grab the specimen. (G) Crushed the specimen in the specimen bag when the cutter head of the pulverizer was mostly hidden in the trocar. (H) Crushed the specimen in the specimen bag when the cutter head of the pulverizer was completely all hidden in the trocar.
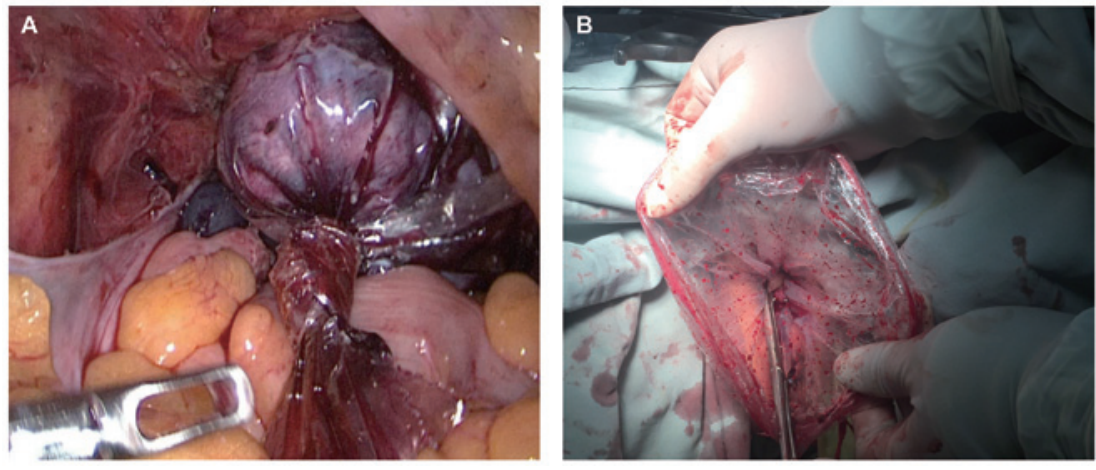

Figure 4. Operation of specimen bag during hysterectomy. (A) Whole uterus specimen was taken from the vagina after being placed in the bag. (B) Specimen bag was taken out of the vagina and the specimen was smashed in the bag. 

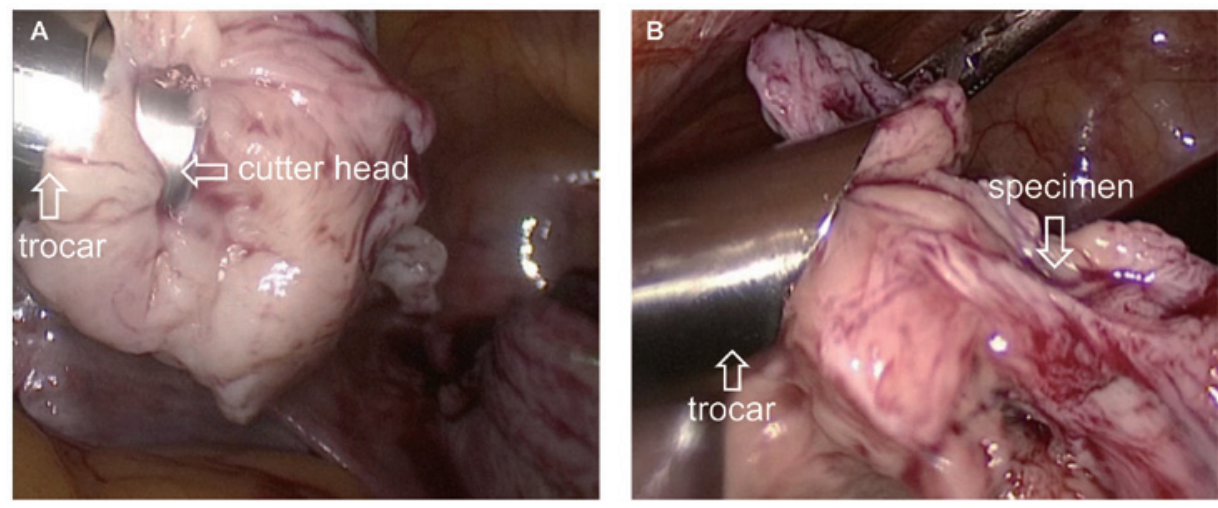

Figure 5. Position of the cutter head. (A) Cutter head was mostly hidden in the trocar. (B) Cutter head of the shredder was completely hidden inside the trocar.

When smashing fibroids, use of the specimen bag prevents small specimen fragments from splashing into the abdominal cavity, causing intra-abdominal implantation and spread. For patients with degenerative uterine fibroids, the bag also avoids the spread of tumor cells that are invisible to the naked eye. It is only necessary to smash the specimen to $<1.5 \mathrm{~cm}$ during surgery (i.e., the size of the incision), which decreases the surgical time; finally, the small specimen is removed from the abdominal cavity together with the specimen bag. No small fibroid tissue remains in the abdominal cavity. When the uterus is comminuted in the vagina during total hysterectomy, the protection of the specimen bag prevents implantation of the tumor including the endometrium in the abdominal cavity. In the present study, no damage to the intraoperative specimen bag occurred in any patient, and the abdominal wall wounds healed after surgery.

Outcomes following the laparoscopic myomectomy group. In the laparoscopic myomectomy group, the data analysis showed no significant differences in the preoperative data between the specimen bag group and the non-standard bag group $(\mathrm{P}>0.05)$. The diameters of the uterine fibroids were not significantly different between the groups ( $\mathrm{P}>0.05$; Table I). There was no significant difference in the operation time, intraoperative blood loss, or average hospitalization duration between the two groups ( $\mathrm{P}>0.05$; Table II). These findings indicate that intraoperative use of the specimen bag is a feasible procedure. The follow-up ranged from 1 to 23 months (average, 12.6 months), and the follow-up rate was $100 \%$. No postoperative complications such as wound infection or bleeding occurred.

Outcomes following the laparoscopic hysterectomy group. In the laparoscopic hysterectomy group, the uterus body was smashed and taken out of the vaginal opening under direct vision. Five patients had hematuria because of the uterine was so large that the intravaginal specimen was taken longer during the operation. Hematuria disappeared by $24 \mathrm{~h}$ postoperatively, and the urethral catheter was removed 3 days postoperatively; no urinary leakage occurred. The average time for specimen bag placement was $4.42 \mathrm{~min}$, which was negligible. No rupture of the specimen bag occurred during the operation, no complications occurred after the operation, and the average length of hospital stay was within the plan.
Table V. Specific surgical data of the specimen bag group in laparoscopic hysterectomy.

\begin{tabular}{lc}
\hline Parameter & Surgical data \\
\hline Average uterus weight, $\mathrm{g}$ & $372.31(210-710)$ \\
Uterine weight $\geq 300 \mathrm{~g}, \mathrm{n}$ & $32(65.31 \%)$ \\
Place specimen bag time, min & 4.47 \\
Intraoperative complications, $\mathrm{n}$ & $5(10.20 \%)$ \\
Intraoperative specimen bag rupture, $\mathrm{n}$ & $0(0 \%)$ \\
Transfer to open, $\mathrm{n}$ & $0(0 \%)$ \\
Postoperative complications, $\mathrm{n}$ & $0(0 \%)$ \\
Average hospital stays, days & $4.76(4-6)$
\end{tabular}

Postoperative follow-up was performed for 1 to 23 months (average, 11.3 months). The follow-up rate was $100 \%$. No complications such as wound infection, hemorrhage, or urinary tract injury occurred (Table V).

\section{Discussion}

Traditional open surgery, specimens can be easily removed from the abdominal cavity. However, in laparoscopic myomectomy and laparoscopic hysterectomy, the specimen is too large to be directly removed from the abdominal wall incision or the vagina. In 1991, Semm reported the first manual crushing device (5). In 1993, Steiner et al invented the electric splitter (6). The US Food and Drug Administration (FDA) reviewed and passed the use of an electric splitter in myomectomy (K946147) and total hysterectomy (K993801) in 1995 and 2000, respectively, which undoubtedly promoted the rapid development of gynecological laparoscopy. However, many clinical data analyses have shown that the use of electric shredders can actually increase the dissemination of uterine fibroids $(7,8)$, while the dissemination rate of uterine sarcoma after abdominal dissection is as high as 13 to $57 \%(7,9,10)$. Park et al (4) retrospectively analyzed 56 cases of uterine leiomyosarcoma, 25 of which had involved the use of electric shredders. They found that use of the electric shredder was associated with a worse prognosis and shorter survival time. Morice et al (11) reported that the recurrence and metastasis rates in the pelvic and abdominal cavities increased within 3 months after malignant tumor tissue was divided. At 
the end of 2014, the FDA issued a statement on the use of electric breakers in minimally invasive surgery for uterine fibroids, indicating that the use of shredders in patients with suspected or clear uterine malignancies should be avoided (12). So some scholars have suggested abandoning use of the shredder (13). The literature indicates that the incidence of uterine fibroid sarcoma is approximately 0.03 to $1.00 \%$ (14). Although imaging techniques have been greatly improved, the differential diagnosis of uterine fibroids and uterine sarcoma before surgery is difficult, and diagnostic criteria for preoperative suspicious leiomyosarcoma are still lacking. Therefore, diagnosis of uterine malignant tumors is still often made after pathological diagnosis of uterine fibroids, which leads to intraoperative tumor dissemination. Even in patients with benign uterine leiomyoma, peritoneal disseminated leiomyoma may occur. However, when a pulverizer is applied to the whole uterus, it may cause endometrial and malignancy dissemination. In this way, minimally invasive surgery has become extremely important.

To this end, we considered placing the specimen in a sealed specimen bag for improved comminution. Although specialized closed specimen bags are commercially available, but they are expensive, and increase not only the patient's economic burden but also the hospital's expenditure on medical resources. We modified the disposable luminal sheath in the basic configuration of the laparoscope into a specimen bag. The specimen bag is free to the patient. For the medical institution, the material of the specimen bag is a necessary material for the endoscope, even in primary hospitals. The low price of this material will not significantly increase the medical expenses. Notably, however, the improved smashing of the uterus specimen is associated with limited vision, which makes the operation more difficult and indirectly prolongs the operation time. If we can improve the material of the specimen bag and make the visual field high, we can solve this problem.

To resolve the above difficulties and better avoid tumor spread, the author recommends the following based on clinical experience (1). When laparoscopic uterine fibroids are removed, the specimen bag is placed before the fibroids are removed (2). The incision on the surface of the uterine fibroids should be large enough to facilitate removal of the fibroids, and the removal of fissures during the process of removing the fibroids should be minimized. This is also beneficial to reduce the spread of tumor cells during the process of stripping the fibroids (3). After the fibroids have been removed, the specimens are put directly in the specimen bag that has been pre-placed in the uterine rectal sulcus to reduce the exposure time of the fibroids in the abdominal cavity (4). The uterus was sutured before the specimen was taken. This not only reduces the bleeding of the wound surface but also slightly reduces the volume of the in vitro specimen, which is conducive to clear vision when crushing (5). The specimen bag should be large enough, and its length should be larger than the diameter of the specimen by 5 to $10 \mathrm{~cm}$. When the fibroids are smashed, the specimen bag is large enough to be inflated, and an adequate length of the specimen bag outside the abdominal cavity is also guaranteed. When the whole uterus specimen is removed from the vagina, it is necessary to ensure that the mouth of the specimen bag is located outside the vaginal opening to fully protect the vagina and the abdominal cavity (6). When smashing the fibroids, the specimen bag is preheated with warm water before being placed in the abdominal cavity to reduce the fog generated in the intraoperative bag. Some surgeons can master intraoperative use of a mirror, and sometimes the mirror can even be attached to the specimen bag to observe the specimen in the bag (7). When smashing fibroids, it is necessary to master the use of a pulverizer. The trocar of the pulverizer should be placed in the center of the specimen bag, and the cutter head should be located approximately $5 \mathrm{~cm}$ below the abdominal wall. The cutter head of the pulverizer can be mostly or even all hiddend in the trocar, which prevents the specimen from rotating with the cutter head and prevents damage to the surrounding organs by the cutter head (Fig. 5). When pulverizing the fibroids, the surgeon should start from the surface; the inside of the fibroids should not be penetrated, and good vision and surgical safety should be ensured. Specimens that are pulverized to a diameter of $\leq 1.5 \mathrm{~cm}$ can be removed without being taken out together with the specimen bag, and the time for pulverizing the specimen is reduced.

Our hospital has improved the laparoscopic uterine smashing technique, which has been applied to laparoscopic uterine fibroid removal in 42 cases, laparoscopic uterine double salpingectomy in 43 cases, and laparoscopic uterine bilateral adnexectomy in 6 cases. Among these 91 cases, none were abandoned due to accidents. During laparoscopic myomectomy, creation and insertion of the specimen bag took a short time, and use of the specimen bag reduced the time required for smashing and removal of the specimen debris; thus, the operation time was not significantly extended. During laparoscopic hysterectomy, when the specimen is too large to be removed from the vagina at one time, we have adopted an improved method that only increases the time to place the specimen bag (average time of $4.47 \mathrm{~min}$ ) for the entire procedure. This increase is negligible in terms of the entire operation. No signs of tumor dissemination were observed throughout follow-up in the present study. However, the follow-up time was limited, and the postoperative pathologic examination confirmed that the data of malignant tumors was too little. Thus, longer-term follow-up and additional samples are needed. Additionally, the specimen bag can be used for the laparoscopic removal of ovarian tumors. Because the pulverizer is not involved, it does not need to be inflated and can be directly removed from the abdominal cavity or vagina. During the removal process, the ovarian tumor can be aspirated and drained in the bag to capture the specimen. Moreover, our self-made specimen bags are made from disposable laparoscopic sheaths. Even in primary hospitals, such sheaths will be used as long as the endoscopes are used. This product is inexpensive, does not incur an extra charge, and does not significantly increase the medical costs. This can be described as a 'win-win situation.' The herein-described self-made specimen bag technology is suitable for a wide range of applications, especially in primary hospitals.

\section{Acknowledgements}

Not applicable.

\section{Funding}

The present study was supported by a grant from The Science and Technology Project of Jiaxing in China (grant no. 2018AD32061; patent no. 2018206067047). 


\section{Availability of data and materials}

The datasets generated and analyzed during the current study are available from the corresponding author on reasonable request.

\section{Authors' contributions}

XS and SZ conceived the study and conducted a critical pre-submission review of the manuscript. XS was responsible for patients' enrollment, operations practice and the manuscript writing. LS made substantial contributions to the design of the study. XS, LS and SZ scanned and identified the relevant literature according to the inclusion and exclusion criteria. XS, LS and SZ and modified parts of the manuscript. All the authors have read and approved the final version of the manuscript for publication.

\section{Ethics approval and consent to participate}

All patients provided written informed consent prior to enrolment. The present study protocol was approved by the institutional The First Affiliated Hospital of Jiaxing University (2017-192). The procedures followed were in accordance with the ethical standards of the responsible committee on human experimentation (institutional or regional) and with the Helsinki Declaration of 1975, as revised in 2000.

\section{Patient consent for publication}

All patients approved the publication of the present study.

\section{Competing interests}

The authors declare that they have no competing interests.

\section{References}

1. Ryan GL, Syrop CH and Van Voorhis BJ: Role, epidemiology, and natural history of benign uterine mass lesions. Clin Obstet Gynecol 48: 312-324, 2005.
2. Wallach EE and Vlahos NF: Uterine myomas: An overview of development, clinical features, and management. Obstet Gynecol 104: 393-406, 2004.

3. Hagemann IS, Hagemann AR, LiVolsi VA, Montone KT and Chu CS: Risk of occult malignancy in morcellated hysterectomy: A case series. Int J Gynecol Pathol 30: 476-483, 2011.

4. Park JY, Park SK, Kim DY, Kim JH, Kim YM, Kim YT and Nam JH: The impact of tumor morcellation during surgery on the prognosis of patients with apparently early uterine leiomyosarcoma. Gynecol Oncol 122: 255-259, 2011.

5. Semm K: Hysterectomy via laparotomy or pelviscopy. A new CASH method without colpotomy. Geburtshilfe Frauenheilkd 51: 996-1003, 1991 (In German).

6. Steiner RA, Wight E, Tadir Y and Haller U: Electrical cutting device for laparoscopic removal of tissue from the abdominal cavity. Obstet Gynecol 81: 471-474, 1993.

7. Tan-Kim J, Hartzell KA, Reinsch CS, O'Day CH, Kennedy JS Menefee SA and Harrison TA: Uterine sarcomas and parasitic myomas after laparoscopic hysterectomy with power morcellation. Am J Obstet Gynecol 212: 594.e1-10, 2015.

8. Bogani G, Cliby WA and Aletti GD: Impact of morcellation on survival outcomes of patients with unexpected uterine leiomyosarcoma: A systematic review and meta-analysis. Gynecol Oncol 137: 167-172, 2015

9. Ehdaivand S, Simon RA, Sung CJ, Steinhoff MM, Lawrence WD and Quddus MR: Incidental gynecologic neoplasms in morcellated uterine specimens: A case series with follow-up. Hum Pathol 45: 2311-2317, 2014.

10. Liu FW, Galvan-Turner VB, Pfaendler KS, Longoria TC and Bristow RE: A critical assessment of morcellation and its impact on gynecologic surgery and the limitations of the existing literature. Am J Obstet Gynecol 212: 717-724, 2015.

11. Morice P, Rodriguez A, Rey A, Pautier P, Atallah D, Genestie C, Pomel C, Lhommé C, Haie-Meder C, Duvillard P and Castaigne D: Prognostic value of initial surgical procedure for patients with uterine sarcoma: Analysis of 123 patients. Eur J Gynaecol Oncol 24: 237-240, 2003.

12. Hampton T: Use of morcellation to remove fibroids scrutinized at FDA hearings. JAMA 312: 588, 2014.

13. Kho KA and Nezhat CH: Evaluating the risks of electric uterine morcellation. JAMA 311: 905-906, 2014.

14. Sala E, Rockall AG, Freeman SJ, Mitchell DG and Reinhold C: The added role of MR imaging in treatment stratification of patients with gynecologic malignancies: What the radiologist needs to know. Radiology 266: 717-740, 2013. 